\title{
ESTADO DE LA INVESTIGACIÓN: LOS TALLERES DEL MUNDO GLOBAL, LA MAQUILA TEXTIL Y LOS CALL CENTERS EN CENTROAMÉRICA
}

\section{RESEARCH STATUS: THE WORKSHOPS OF THE GLOBAL WORLD, TEXTILE MAQUILA AND CALL CENTERS IN CENTRAL AMERICA}

\author{
Nancy Piedra Guillén*
}

\section{RESUMEN}

Este artículo presenta un balance crítico de las investigaciones que se han realizado en las últimas tres décadas sobre el tema de las transformaciones en el mundo del trabajo en el sector de la maquila textil y los "call centers" o centros de llamadas. Principalmente, temáticas sobre las condiciones laborales y de empleo de quienes trabajan en estos lugares en Costa Rica. El proyecto enfatiza en estos sectores de actividad por considerar que ambos ejemplifican claramente el proceso de globalización del cual formamos parte actualmente $y$ se evidencian las consecuencias que se derivan de dicho modelo económico. Pues mientras la maquila textil forma parte de las nuevas formas de trabajo del final del siglo xx, ancladas en la lógica de las cadenas de producción; los "call centers" corresponden al proceso de tercerización de la producción en el siglo xxI, asentados en la promoción de servicios por contratación, contribuyen (do) al desarrollo de la terciarización de la economía del país.

PALABRAS CLAVE: COSTA RICA * TRABAJO * CENTROS DE LLAMADAS * INDUSTRIA TEXTIL * GLOBALIZACIÓN

\section{ABSTRACT}

This article presents a critical balance of the research that has been carried out in the last three decades on the subject of work (i.e. labor); specifically those studies that analyze the transformations in the world of work, to work in the textile "maquila" sector and call centers. Our literature review focuses on these two economic sectors because it responds to a research project whose theme revolves around the working conditions and employment of people working in the textile "maquila" sector and call centers. This focus considers both as two clear examples of socioeconomic activities that respond

Escuela de Sociología y Posgrado Centroamericano en Sociología, Universidad de Costa Rica, Montes de Oca, San José, Costa Rica.

npiedraguillen@yahoo.com/nancy.piedra@ucr.ac.cr 
to the process of globalization of which we are a part today and live the consequences derived from this socioeconomic model. The textile "maquila" is part of new forms of work which originated at the end of the 20th century, anchored in the logic of production chains, while call centers are part of the process of outsourcing production in the 21st century, based on the promotion of contracting services. Both sectors contribute to the development of the tertiarization of the economy in the Costa Rica.

KEYWORDS: COSTA RICA * LABOUR * CALL CENTER * TEXTILE FACTORY * GLOBALIZATION

\section{INTRODUCCIÓN}

Así como la sociedad y el sistema capitalista se han modificado a lo largo de la historia, en el mundo del trabajo se están generando transformaciones tecnológicas, económicas, organizativas, espaciales, sociales, políticas y culturales (Reygadas, 2011), las cuales impactan y afectan a las personas (Mora, 2011). El trabajo en las últimas décadas ha tendido a la desregulación, la flexibilización y la precariedad laboral. Como consecuencia, la incertidumbre es un estado mental que influye más en la actualidad $y$ forma parte de la cotidianidad para un gran sector de la población.

La desregulación de las relaciones laborales y la consecuente disminución de la protección del trabajo tienen la finalidad de aminorar los costos empresariales, incrementar las ganancias para las compañías e incentivar la inversión y la captación de inversiones extranjeras. La maquila textil y los call centers son dos ejemplos que responden a procesos de globalización, a pesar de la primera forma parte de las nuevas formas de trabajo del final del siglo xx, ancladas en la lógica de las cadenas de producción; los call centers forman parte del proceso de tercerización de la producción del siglo xxI, asentados en la promoción de servicios por contratación. Ambos se generan a partir del modelo económico neoliberal, desplegándose un nuevo entorno sociolaboral en la región latinoamericana y el mundo en general, que ha marcado una particular y creciente heterogeneidad en los mercados de trabajo $y$ un notable deterioro de las condiciones de vida de la población trabajadora. Si las condiciones de trabajo y empleo se han deteriorado, no cabe duda de que lo mismo ha ocurrido en la vida cotidiana de las personas. Pocas veces se observa la relación que existe entre ambos ámbitos, pero la vida de las personas no se produce y reproduce solo desde el trabajo productivo, sino que también se agencia desde el mundo íntimo, familiar $y$ social (Hochschild, 2007a, 2007b, 2008).

El presente artículo forma parte de una investigación que se ha estado desarrollando sobre los procesos de transformación laboral y las actuales condiciones de empleo y trabajo ${ }^{1}$, así como la incidencia de ambas esferas en la vida de las personas, considerando temáticas como el valor del trabajo, la vivencia de su temporalidad, los efectos en la salud $y$ las diferencias de género. Siendo ese el interés, en este artículo se enfatiza en la naturaleza de los estudios que se han realizado en las últimas tres décadas, con el fin de conocer sistemáticamente la literatura existente $y$, con ello, retroalimentar el análisis empírico.

El documento se estructura en las siguientes secciones: primero, se analizan los procesos de transformación en el mundo del trabajo; posteriormente, se reseñan algunos estudios que abordan la presencia de las mujeres en el mercado laboral como un fenómeno creciente en la región latinoamericana y luego, se refiere a los estudios que se han realizado a nivel nacional sobre la maquila textil, por ser tema de interés en la investigación propuesta, para finalmente, caracterizar los estudios que se han realizado sobre los call centers.

$1 \quad$ El nombre del Proyecto en la Vicerrectoría de Investigación es: Pry01-1128-2018 "De la maquila textil a la maquila de los servicios: análisis comparativo de las condiciones de empleo, trabajo $y$ vida cotidiana de las y los trabajadores de la industria textil y los call centers". 
PROCESOS DE TRANSFORMACIÓN EN EL MUNDO DEL TRABAJO Y SU ANÁLISIS

Se considera inicialmente que, en lo relativo a la temática laboral, en América Latina predominaron, hasta las décadas del 80 y 90 del siglo pasado, los estudios relacionados con el movimiento obrero. Este énfasis predominó por el contexto social, caracterizado por las dictaduras militares en países como Argentina, Chile, Uruguay, Brasil, Bolivia Ecuador; en los que hubo una fuerte presencia de los sindicatos como agentes que luchaban por la democratización de los procesos políticos y defensa de los derechos laborales. Académicos como Touraine, Torcuato Di Tella y E.P. Thompson fueron considerados un referente intelectual, pero muy moderados para el contexto latinoamericano. Es a finales de la década de los 80 que surgen otros enfoques como parte de los Nuevos Estudios Laborales, el cual tuvo origen en nuestra América Latina antes que los fenómenos de reestructuración productiva de los 80 , asociados a los procesos de urbanización e industrialización en Latinoamérica: "Todas las vertientes de los Nuevos Estudios Laborales sufrieron el impacto de la emergencia del nuevo modelo económico neoliberal, la reestructuración productiva en grandes empresas y sus consecuencias en mercados de trabajo, condiciones de trabajo $y$ relaciones laborales" (Baglioni, 1987; Baglioni y Crouch, 1990, como se citaron en de la Garza, 2016).

Enrique de la Garza (2015) señala que, como ocurrió en Europa, con la generación de los nuevos estudios laborales en México se desarrolla la perspectiva socio demográfica para el estudio de los mercados de trabajo. Dicho análisis introduce el concepto de marginalidad, así como el de informalidad, donde se incorporan variables explicativas como las de género, edad, escolaridad, tamaño de la familia y estado civil. Actualmente, estos son aspectos que se consideran en las encuestas de empleo.

Con base en estos estudios, surgen conceptos influyentes en la interpretación sociológica, como las estrategias de sobrevivencia, de vida, discriminación, segregación sociolaboral. A partir de los datos obtenidos se conformó una sociodemografía del mercado de trabajo en México. De igual modo, en otros países de América Latina se generan estudios similares, $y$ los conceptos mencionados se convierten en una referencia para comprender los fenómenos socioeconómicos. En 1993, surgen los estudios de la nueva institucionalidad de la reestructuración productiva, $y$ sigue con fuerza la sociodemografía. Esta perspectiva sociodemográfica fue más descriptiva y menos teórica; sin embargo, tiene como punto de partida la teoría de la dependencia, en donde el concepto de marginalidad fue medular, pues llevó a discutir la informalidad en el trabajo. Esta se entendía como la entrada y la salida de la mano de obra que, en su mayoría, atravesaba procesos de migración campo-ciudad. Por otra parte, el análisis de las lógicas productivas tendió hacia la reproducción de la vida social, es decir, de la familia como unidad productiva central (económica y social). De allí se desprenden dos conceptos importantes: "estrategias de vida" y "estrategias de sobrevivencia” (de la Garza, 2007).

Entre 1993 y el 2000 es la época del oro de los nuevos estudios laborales y hay logros en la reflexión y en la investigación empírica. Por ejemplo, en México se crea un mapa de las transformaciones nacionales de los contratos colectivos y la tendencia es hacia la flexibilidad laboral, con lo cual se aporta un análisis de los nuevos sujetos ${ }^{2}$ obreros en las industrias reestructuradas. Hay reelaboración de las teorías

2 En otro texto, de la Garza (2005) reflexiona sobre los límites que tiene el concepto de trabajo desde dos visiones, una neoclásica y otra marxista, $y$ de cómo ello incide en la construcción de la subjetividad de los propios trabajadores $y$, al mismo tiempo, en la construcción de movimientos sociales laborales. De la Garza introduce la idea del sujeto consumista dentro del análisis de la sociología del trabajo, $y$, en general, como parte esencial del engranaje capitalista, que en las relaciones de producción y de acumulación es un elemento fundamental, y es bajo la concepción del consumo que también se crean y legitiman las desigualdades sociales. Es decir, el sujeto puede tener esta doble connotación (trabajador y consumidor), y en el consumo existen relaciones de poder. Además, de la Garza sostiene que el trabajo lleva implícito relaciones sociales amplias de diversos tipos: "de poder, de interés, de influencia, culturales" (de la Garza, 2005, p. 13). 
de la reestructuración, que no se reducen al postfordismo, $y$ hay una preocupación sobre cómo se vinculan las subjetividades con las estructuras explicando el tema del control sobre el trabajo (de la Garza, 2015).

Se inicia una gran polémica interna entre los optimistas y los pesimistas. Los primeros, retoman la cara amable del postfordismo, de la regulación y especialización flexible; los segundos, plantean el término de "límites de la reestructuración productiva", "el impulso de vías salvajes" y la introducción del concepto de "crisis del toyotismo", porque no da más de sí para aumentar la productividad. Además, imponen las vías unilaterales, salvajes, autoritarias como estrategias de las empresas que predominan en México y otros países de América Latina. Es decir, lo que predomina como organización del trabajo es una especie de toyotismo precario, como las formas de trabajo en equipo y los círculos de calidad que precarizan a las personas trabajadoras. Los regulacionistas, por su parte, centran su análisis en las instituciones y los sujetos quedan de lado. Con esto, imperan la perspectiva estructuralista, pero se genera una perspectiva crítica a la lógica postfordista.

Iniciada la década del 80, a la transformación estructural se le suman cambios en el mundo académico, se está ante una "crisis" relativa del marxismo, agotamiento del dependentismo y surgimiento de una nueva generación de investigadores e investigadoras que brindan las condiciones necesarias para el repunte de nuevos estudios del trabajo en América Latina. Estos estudios adoptaron marcos teóricos amplios y diversos, entre los cuales se destaca el Enfoque de la Regulación ${ }^{3}$,

3 Para la vertiente de estudio de los procesos de trabajo - que es ver el trabajo como actividad y no como situación, como lo había planteado Touraine (1988) —, el parteaguas desde el punto de vista de los marcos teóricos significó el estudio y la incorporación durante la década de los 80 o a inicios de los 90 de las llamadas Teorías del postfordismoRegulacionismo francés (Boyer, 1989), la de la Especialización Flexible (Piore y Sabel, 1990) y las neoschumpeterianas (Peres y Ominami, comp., 1985) en sus primeras formas. Hay que aclarar que la influencia directa de las teorías de sociología del trabajo, como la escuela francesa que partió basado en los trabajos de Aglietta (1976), Boyer (1996), Coriat (1993), Lipietz (1983, 1992), entre otros. Este enfoque pretendió sustituir la teoría de la dependencia. También se reconoce el surgimiento de teorías sobre el toyotismo y el "lean production", los Nuevos Conceptos de Producción de Kerr y Schumann (1984; 1998), la especialización flexible de Michel Piore y Charles Sabel (1990), la teoría del "industrial governance" y su preocupación sobre las cadenas productivas, entre otras.

A finales de la década de los 90, surge el concepto de la identidad en el trabajo, se pasa de cultura laboral al problema de la identidad. El análisis de Bauman (2004) retoma esta reflexión que desde su lógica de la fragmentación de las identidades, como una de las corrientes que plantea el tema del fin del trabajo, su objetivo es demostrar que ya no hay identidades.

El siglo XXI trajo nuevas perspectivas a los enfoques sobre el trabajo, por ejemplo, se estudia temas como el valor del trabajo, la experiencia subjetiva y la construcción de identidad, con ello se amplía la lógica dominante en la que se estudiaba enfáticamente las condiciones de trabajo. Los optimistas se inclinan por el estudio de la manufactura y la maquila, por lo que abundan textos sobre dicha actividad. Estos enfatizan en el cambio del ensamble sencillo a las nuevas formas de organización y luego a la creación de conocimiento, reforzando con ello, esa perspectiva optimista del trabajo y la nueva división internacional de este. Aun así, el debate empírico se centra en mostrar la forma en que las empresas maquiladoras no siguen el camino de la producción del conocimiento, sino lo contrario: se genera trabajo precario, bajo el valor agregado, con un proceso de producción fragmentado, insumos que siguen siendo importados con una mano de obra que no ha cambiado,

de Friedman, Naville o Touraine, o bien, la sajona de Braverman (1974), así como las de relaciones laborales a la manera de Dunlop, Goldthorpe (1989), Hyman $(1987,1996)$ y otros, en el arranque de estos estudios no fueron las más influyentes, a pesar de que hubo intentos limitados de incorporarlas a la discusión desde los 70, especialmente en las de corte obreristas (Negri, 1978; Mallet, 1972; Panzieri, 1978), aunque también funcionalistas (de la Garza, 2016). 
pues son procesos de ensamble y los sindicatos (que deberían protegerlos) cuando los hay, son ficticios (de la Garza, 2015).

Por su parte, la visión de los pesimistas también deviene de la corriente que enfatiza el fin del trabajo desde la teoría de la innovación sin trabajo. Es decir, el trabajo no importa, la innovación se hace de otra manera. Desde esta perspectiva, se pasa a estudiar temas consistentes en la innovación sin trabajo.

La reflexión teórica ha sido muy rica al respecto, lo que lleva a varios estudiosos y estudiosas a plantearse el tema de los trabajos no clásicos. Se introduce la noción del cliente como parte del trabajo, $y$ se establece la idea de la triada "cliente, persona empleadora y persona trabajadora" como una relación intrínseca de la actividad que se desarrolla, en la que se establece una triada diferente a la de décadas atrás en la cual lo importante era la relación del Estado, empleador/a y trabajador/a. El tema de la subjetividad y la identidad se replantea desde otra perspectiva.

Los análisis sociológicos del mundo del trabajo se multiplican y "obligan a reconstruir marcos teóricos de la conciencia obrera, de la acción sindical y también de las relaciones de las y los trabajadores organizados con las instituciones laborales y los sistemas políticos" (Zapata, 2010, p. 17). Es necesaria la construcción de esquemas de interpretación para analizar sectores económicos, empresas y tipos de personas trabajadoras. Por ello, se inclina a pensar $y$ coincidir con quienes consideran que los estudios de caso, las entrevistas en profundidad o el uso de métodos similares, son más pertinentes para estudiar lo que sucede en el mundo del trabajo, puesto que se observa la heterogeneidad, así como a las trabajadoras y a los trabajadores como personas y como sujetos sociales.

En Centro América, el tema de las maquilas -industria textil transnacional- fue un tópico de interés regional principalmente en la década de los 90 del siglo xx. Actualmente es un tópico poco tratado en el país, pero de auge en la región latinoamericana. La actual crisis económica define un nuevo panorama: a pesar de que surge en el sistema financiero, se expande $y$ afecta al sistema productivo e impacta negativamente la oferta de trabajo. Como consecuencia, se generan altas tasas de desempleo y aumentan la inseguridad, la precarización y la flexibilidad (contratos de corta duración, contratos a destajo, contratos más verbales que escritos, etc.) (Mora, 2008). Si bien esto es cierto, también se debe de hacer notar que el fenómeno descrito "no es homogéneo, no implica a todos los sectores económicos ni a todas las categorías de trabajadores" (Zapata, 2010); sin embargo, describe la realidad de los sectores de estudio para esta ocasión.

Como se indica anteriormente, a partir de la década de los 70 y 80 , se originan nuevos modelos de organización del trabajo que plantean innovaciones absolutas o reformulaciones de los viejos modelos: el toyotismo, hondismo, la "Lean Production", "Industrial Governan$c e "$, entre otras. En cuanto al toyotismo, se identifica una fuerte ruptura con el modelo taylorista-fordista. Antunes (2003) señala que "la producción bajo el toyotismo está orientada directamente por la demanda. La producción es variada, diversificada y capaz de abastecer el consumo. Es éste el que determina lo que será producido, a diferencia de cómo es en la producción en serie y en masa del fordismo" (p. 30). Algunas de sus características fueron la superación del trabajo parcelado, se promueve el trabajo en equipo, el esfuerzo suele a ser colectivo, el trabajo individual se complementa con el colectivo. Esto supuso una mayor flexibilidad en la organización de la fuerza de trabajo. En términos de contratación, hizo que, por ejemplo, se contase con un mínimo de trabajadores a tiempo completo o se realizasen subcontrataciones, así como contrataciones parciales y temporales, además de un uso intensivo de las tecnologías de la información y comunicación. Por otro lado, se redujeron los costos de producción, se aumentó hasta lo justo la producción, y se disminuyeron los riesgos potenciales (como la organización sindical) por medio de dos tácticas generalizadas: la subcontratación de empresas y la deslocalización geográfica (Antunes, 2003).

El enfoque de la regulación permite comprender el funcionamiento estructural y global de un sistema social, cómo se 
estructuran las condiciones económicas de forma asociada a una configuración particular de relaciones sociales, tanto a nivel institucional formal e informal (regulación) $y$ de las relaciones en el proceso de producción de riqueza (organización del trabajo). Dicho, en otros términos, el enfoque permite analizar "la conjunción de mecanismos que concurren a la reproducción del sistema en su conjunto, dadas las formas institucionales en vigor y el estado de las estructuras económicas y sociales" (Boyer (s.f.) como se citó en Neffa, 2005, p.9).

En el año 2010, de la Garza y Neffa presentaron una obra que constituye una de las investigaciones de mayor relevancia en la última década sobre el tema de los modelos de trabajo. Con la participación de más de 10 investigadores, el estudio plantea una discusión teórica sobre el tema de los modelos económicos, modelos productivos y estrategias de ganancias en el contexto latinoamericano postcrisis financiera del 2008. De esta forma, se realiza un repaso de estudios de caso sobre el tema, entre los cuales se aborda México, Colombia, Brasil, Argentina y Venezuela. A nivel teórico, de la Garza y Neffa (2010) problematizan el concepto de modelo productivo a partir de las elaboraciones de Boyer y Freyssenet (2003), quienes señalan que, debido a que la economía pensó al individuo sin considerar otras ciencias sociales, la economía se configuró como una disciplina meramente utilitaria en busca de lo óptimo sin tomar en cuenta otras esferas de la vida social: "las abstracciones acerca de cómo considerar la acción económica también deberían relacionarse con la forma de definir el problema" (p. 56). Estos autores lo plantean como una "consecuencia" epistemológica (de la Garza y Neffa, 2010 y de La Garza, s.f.).

Desde la perspectiva teórica, se observa a finales del siglo xx, el (re) surgimiento del neoinstitucionalismo, que es propiciado por la sociología y la economía en conjunto, en cuanto se vinculan los conceptos de actor racional $y$ acción económica.

De la Garza (s.f.; 2007; 2010) identifica los postulados de esta corriente según la disciplina. En la economía, refiere a la idea de buscar reducir, en la medida de lo posible, los costos asociados a las transacciones mercantiles (a través de los mercados o jerarquías de empresa); esto se basa en una lógica de demanda, oferta y precios. Luego, se afirma que "las instituciones pueden proveer soluciones a las fallas del mercado" (p. 34). En este caso, la organización empresarial puede limitar la racionalidad.

En el caso de la Sociología, el neoinstitucionalismo surge de las inconformidades teóricas con el holismo planteado por Talcott Parsons: "los sistemas de reglas estructuran la acción y limitan la capacidad de alcanzar óptimos." (de la Garza, s.f.: 35). Estas normas se traducen en la institucionalidad social que limitan la libertad de ejecutar acciones racionales.

En segundo lugar, aparecen las teorías que de la Garza (2016) contrasta con sus elementos centrales:

1) Industrial Governance: instituciones y sujetos coordinan las dinámicas de los actores económicos, en donde el mercado es la red de relaciones formales e informales. Aquí es más importante el poder que los precios, los individuos poseen racionalidad limitada y las instituciones son sistemas de reglas.

2) Modelos productivos: el mercado es una construcción social con constantes regulaciones impuestas que son externas a él, las tasas de ganancia provenientes de la producción no son las óptimas sino satisfactorias, ya que están sujetas a límites cognitivos, de cálculo, al contexto, $y$ a las estrategias de los actores.

En tercer lugar, recupera una serie de constructos teóricos pertinentes:

1) Context bounded rationality: la racionalidad limitada por restricciones institucionales.

2) Racionalidad emergente: lo que orienta la acción del individuo es el cúmulo de otras experiencias similares que han sido exitosas.

3) Embeddedness: es el carácter "enredado" dentro de la acción social, y que está condicionada por un engranaje institucional 
o por redes sociales (existe un debate entre autores en torno a esto).

De la Garza $(2007 ; 2010)$ muestra que en los nuevos estudios sobre relaciones laborales el concepto que sigue predominando es el de flexibilización. Sin embargo, desde una perspectiva neoliberal, se busca la desregulación en el mercado de trabajo para alcanzar más eficiencia. Específicamente, se procura implementar nuevas leyes laborales o transformar las existentes que se enfoquen en aspectos de subcontratación, descentralización de contratación colectiva, extensión de períodos de contrato sin obligaciones para el patrono, disminución de las indemnizaciones por despido, promoción de los bonos por productividad, entre otros.

En cuanto a las investigaciones sobre subcontratación, los constructos teóricos más importantes son el de producción en red o empresa red e "industrial governance". Asimismo, se reconocen tres tipos de subcontratación o tercerización laboral, con el predominio en América Latina de la llamada red jerárquica, en la cual, las relaciones son unilaterales, muchas veces impuestas, sin mayores beneficios para las personas trabajadoras y se facilitan las condiciones para que sean las empresas quienes eludan las responsabilidades laborales (de la Garza, 2010).

\section{PRECARIZACIÓN Y PRECARIEDAD LABORAL}

Antunes (2015), por medio del método dialéctico, se da a la tarea de deconstruir los conceptos de precarización y precariedad laboral. En el caso de América Latina, decenas de trabajos hacen referencia a dicho proceso. Tomando las propuestas teóricas de distintos autores, Antunes somete a cuestionamiento los conceptos con el fin de recuperar sus aportes $y$, a la vez, de refutar algunos de sus aspectos, con el propósito de concretar su propuesta, en donde construye teóricamente su idea de precarización y precariedad laboral.

Antunes parte de que la modernización se constituyó como una forma de gubernamentabilidad en los países capitalistas e intentó exportarse a los países periféricos. Esto implicó una restructuración de la producción, sumada a las dinámicas migratorias experimentadas en la mayoría de los países, durante las décadas de 1960 y 1970, así como la deslocalización de la producción, lo cual dio como resultado una serie de procesos de descolectivización y reindividualización de las relaciones sociales que fueron caldo de cultivo para la precarización laboral.

La modernidad fracasa como proyecto emancipatorio, en tanto es dominio de élites en busca de "desarrollo", al conformar intentos fallidos de exportar el proyecto a los países periféricos, y se plantea la necesidad de modernización productiva en América Latina, en donde la explotación y la precariedad laboral fueron parte inherente de los procesos de acumulación.

La crisis del fordismo, la desproletarización del trabajo industrial y la deslocalización de la producción, trajeron consigo la acumulación centralizada y concentrada del capital. Por tanto, era necesario hacerlo a partir de condiciones laborales precarias que explican una de las distintas expresiones de la desigualdad social. La precarización se traduce en la transformación de las relaciones sociales $y$ de las subjetividades e identidades de clase (Antunes, 2014). Esto implica entender la precariedad como temporalidad, pues esta se deriva del proyecto de modernidad y el capitalismo; ambos avanzando a distintas velocidades, en diferentes direcciones y en formas no lineales.

Asimismo, debe distinguirse entre precariedad y precarización; "la primera nace en un escenario donde se construye una América Latina neoliberal y se vincula a insatisfacción, escasez, fragilidad; la segunda hace referencia a un proceso temporal de degradación, pérdida, extensión y prolongación de la falta ordinaria" (Antunes, 2014, p. 158).

Para Antunes (2014), únicamente se puede entender la precarización laboral en su temporalidad. En América Latina es un fenómeno distinto en su génesis que en su actual desarrollo, siempre relacionado a la expansión del neoliberalismo y sus principios. La precariedad es uno de los aspectos "que consolida los procesos de dominación del capital a nivel internacional, en la organización y disposición tecnológica del trabajador." (Antunes, 2014, p. 161). 
Por lo tanto, no es posible generar verdaderos aportes a la discusión de este tema si se realizan análisis deshistorizados, especialmente cuando se trata del contexto latinoamericano. Sin embargo, hay un límite metodológico que debería atenderse con investigaciones que integren el espacio y el tiempo en el que se comprenden las subjetividades-objetividades de las personas trabajadoras propias de esta región.

\section{CONDICIONES DE EMPLEO Y CONDICIONES DE TRABAJO}

Conviene señalar que los estudios realizados en Costa Rica - y en el resto de América Latina- no establecen la diferencia conceptual entre condiciones de empleo y condiciones de trabajo. Comúnmente, estas investigaciones se refieren a las condiciones de trabajo en general $y$ se incluyen dimensiones de análisis $y$ variables haciendo referencia a lo que en este artículo llamamos condiciones de empleo. Debido a que esta diferencia conceptual permite identificar más fácilmente los factores que tienen más impacto en la salud física y psíquica de las personas, se considera dicha perspectiva para el presente trabajo.

Las condiciones de empleo hacen referencia a las circunstancias en las que una persona realiza un trabajo u ocupación. Están constituidas por una serie de garantías, derechos, compromisos y obligaciones implícitas en las diferentes dimensiones e indicadores del trabajo que se establecen formal e informalmente, de manera colectiva o individual, entre el trabajador o trabajadora $y$ la persona empleadora, $y$ que determinan la situación que vive la persona empleada en su trabajo u ocupación. A su vez, estos aspectos pueden alterar o beneficiar en lo individual, familiar y social. Desde una perspectiva general, se analizan variables como el tipo de empleo (fijo, estable o indefinido), horario (completo o parcial), tipo de contrato, calidad del empleo derivado del tipo de contratación y salario. Por su parte, las condiciones de trabajo captan las circunstancias en los centros de trabajo, o bien, en el lugar que se llevan a cabo, determinan la exposición a riesgos en el lugar de trabajo y el modo en que este está organizado (lugar de trabajo y organización del trabajo).
Las condiciones de trabajo materiales incluyen el ambiente físico, químico, biológico y ergonómico, y la organización del trabajo incluye el ambiente psicosocial, la gestión, tareas que se llevan a cabo y el control del trabajo (Benach y Muntaner, 2005; 2009).

Sobre las condiciones de empleo, Pérez (2015) señala que en América Latina el trabajo "no ha devenido suficientemente en empleo $y$, por consiguiente, las relaciones salariales han estado marcadas por una gran asimetría" (p.19). El texto de Pérez Sáinz es un referente para comprender los procesos que se han dado en América Latina en los últimos años, cuyo objetivo es oponerse a los enfoques de inspiración liberal, los cuales, desde su punto de vista, han predominado en la región. Pérez es crítico de los enfoques que surgen de la CEPAL $y$ otros organismos internacionales que enfatizan en las cadenas globales de valor o de producción, ya que estas dejan por fuera las condiciones de trabajo que desde esos ámbitos se generan. Con esto, se enfatiza la dinámica y los nuevos empleos que se generan a nivel mundial a partir de los procesos de apertura económica, social y política (Herzberg, 2010, como se citó en Pérez, 2015).

Para Pérez, esos enfoques devienen en una región latinoamericana cada vez más desigual lo cual se explica, desde su perspectiva, por las condiciones de generación y apropiación de los excedentes económicos. Con ello, se profundizan los procesos de desempoderamiento entre las clases sociales, la etnia/raza y la territorialidad. Pérez retrotrae a un núcleo central del debate y la realidad social, señalando al respecto que "hay que priorizar las clases sociales, porque son los sujetos sociales por antonomasia que disputan el excedente. Si hay excedente hay clases y estas solo existen en su pugna por el excedente" (Pérez, 2015, p. 18). Además, es de suma importancia para el presente estudio destacar que Pérez se distancia de los estudios que no piensan a las personas: "los individuos cuentan y no pueden ser soslayados" (Pérez, 2015).

Ahora, si bien en la práctica se tiende a diferenciar y no a sumar, hay que buscar la homogenización para evitar la fragmentación. En otras palabras, se deben procesar diferencias de distinto tipo como el sexo, el fenotipo, la 
cultura, el territorio, el espacio, entre otros. La construcción social de las diferencias es necesaria para debilitar la oposición de categorías, tales como, género, etnia, raza y territorio: "Si la homogenización es exitosa, la oposición de esas categorías se relativiza y se propician procesos robustos de individuación, que podrán relativizar las dinámicas de clase en los mercados básicos" (Pérez, 2015, p. 18).

\section{SOBRE LA MAQUILA TEXTIL Y LA PARTICIPACIÓN DE LAS MUJERES}

Desde la década del 90, Quintero insistió en la necesidad de estudiar la evolución del sindicalismo maquilador, porque consideraba que este revelaba expresiones diversas, según sea el lugar en el que se observa su actuar. Así, lo que ocurre en las maquiladoras sindicalizadas de Tijuana no es lo mismo que sucede en las de Ciudad Juárez, Reynosa, Piedras Negras o Matamoros (véase Quintero, 1990; 1997; 2006; 2013). Quintero ha desarrollado una tipología en la que distingue entre el sindicato tradicional y el subordinado.

Por su parte, Zúñiga (2005) revisa las disposiciones de la Ley Federal del Trabajo en México y propone que:

... las ausencias que existen en la Ley Federal del Trabajo no sólo se prestan a las interpretaciones $y$ uso restringido de los derechos que encierra, sino que además, favorecen la existencia de prácticas discriminatorias y excluyentes hacia las mujeres, al no incorporar los avances que en materia laboral $y$ de derechos humanos se han suscrito a nivel internacional, contraviniendo con ello los mismos preceptos constitucionales que la sostienen. (Zúñiga, 2005, p. 284).

Este autor concluye, tajante: "la Ley Federal del Trabajo no expresa la realidad laboral que viven las mujeres" (Zúñiga, 2005, p. 285).

En Costa Rica, en la década del 90, se encuentran una serie de investigaciones producidas, en su mayoría, por la Oficina Internacional del Trabajo (OIT) para toda la región centroamericana y del Caribe. En el caso de Costa Rica, se pueden citar los textos de Gitli (1995), Gitly y Vargas (1996), Gitly y Ryd (1992), Gitly (1997), Pérez (1995a y 1995b) como análisis de referencia en los cuales se estudia el comportamiento del sector textil y la cultura laboral en la industria de exportación. Varios de ellos realizan comparaciones entre Costa Rica y otros países de la región (Pérez 1995a y оIT, 1996a) o indagan sobre el giro de la industrialización centroamericana hacia la exportación, proceso incentivado con el crecimiento de las maquilas y las Zonas Francas (Pérez, 1998), así como en las diferencias sobre las condiciones laborales que se pueden encontrar entre las mismas maquiladoras, dependiendo de su origen (Gitli, 1997; Pérez, 1998; Pérez y Cordero, 1996; Carrillo, F., 1999; Cordero, 1999; Carrillo O., 1998). Al respecto, se encontró una marcada diferencia entre las empresas de capital norteamericano y las orientales, al igual que entre las textileras y las que se dedican a la producción de alta tecnología, como en el caso de Intel, Abbot Laboratories, Procter \& Gamble, Western Union, Alcoa CSI, y Remec Inc, entre otras, que se caracterizan por generar empleos de mayor calidad y mejores condiciones laborales (Carrillo, 1999; Cordero, 1999).

En esta línea de estudio, se ubica el texto de Trejos y Abdallah (2000), quienes analizan los procesos de flexibilidad laboral en Centroamérica y destacan la situación de las maquilas debido a las irregularidades que empiezan a observarse en el proceso productivo y en las relaciones laborales. Aquí se generaliza aún más la subcontratación en tareas específicas, con lo que se favorece a las grandes empresas y se precarizan las condiciones labores de las personas. Por otra parte, con respecto a la presencia de las mujeres en las maquilas, se han realizado estudios para América Latina, los cuales muestran que, en distintas ramas de actividad, el empleo femenino se concentra en segmentos productivos secundarios como empresas de menor productividad, menor sofisticación tecnológica u ocupaciones donde se desarrollan actividades repetitivas y precarias. Así, la segregación laboral por condición de género sigue siendo una realidad que afecta la trayectoria $y$ 
los espacios de inserción laboral de las mujeres (Anker, 1997; Abramo, 1999; de la Rica, 2007; Rojo y Tumini, 2008).

Por su parte, también el tema de la participación de las mujeres en la maquila y la migración, o bien, de la movilidad y la precarización del empleo en las textileras transnacionales, han sido objeto de estudio (Grijalva y Covarrubias, 2005; Carrillo y Partida, 2004; Carrillo y Gomis, 2004; De la O y Quintero, 2002). Algunas investigaciones apuntan en esa dirección y tratan temáticas diversas: desde aspectos generales sociodemográficos de las obreras de la maquila y sus condiciones de empleo en general, hasta aquellos que enfatizan en la salud reproductiva en las mujeres, el problema del hostigamiento sexual y la participación sindical de las mujeres.

En esa línea de investigación, se ubica el trabajo de Martínez y Zabalo Arena (2005) quienes analizan el inestable porvenir del sector maquilador en Centroamérica y República Dominicana. Los autores hacen énfasis en la importancia de este sector dentro de estas economías como principal receptor de mano de obra femenina, a pesar de las precarizadas condiciones de trabajo y su escasa remuneración. Desde una perspectiva más cualitativa, se encuentra el informe de investigación de Piedra (2000) que analiza, con un enfoque de género, las condiciones laborales y de vida de las trabajadoras en la maquila textil.

A estos trabajos se unen los estudios de Sandoval (2007) sobre las trabajadoras y los trabajadores de la maquila y la construcción en Costa Rica, donde se destacan las vivencias $y$ condiciones laborales experimentadas. En el trabajo de Florez-Estrada (2007), se analiza la inversión extranjera directa, con particular atención en el Régimen de Zonas Francas. Aunado a esto, Claribel Sánchez (2013) estudia los procesos de precarización laboral del sector de maquila industrial del Régimen de Zona Franca y sus impactos en la vida familiar $y$ doméstica de las mujeres trabajadoras; una de las maquilas está ubicada en el Régimen de Zona Franca del área de mayor desarrollo y la otra, en el área de menor desarrollo. Se puede obsrvar cómo, a pesar de la coincidencia temática entre el trabajo de Sandoval y el de
Sánchez, cada cual enfatiza aspectos distintos, uno en la vivencia y experiencia de las trabajadoras (Sandoval, 2007); la otra, en sus condiciones laborales (Sánchez, 2013), con lo cual ambas investigaciones muestran las adversidades a las que se deben enfrentar las trabajadoras cotidianamente.

Finalmente, los estudios que tratan el tema de la participación de las mujeres en la maquila coinciden en que las temáticas contribuyen en forma clara y contundente a plantear la necesidad de formular una agenda de propuestas para enfrentar la discriminación laboral, el hostigamiento sexual y la violencia latente y manifiesta que sufren las obreras. El presente trabajo introduce también los efectos de dichas condiciones en la salud de las mujeres como cuerpos afectados física y psíquicamente.

\section{LA TEMPORALIDAD EN LOS CALL CENTERS}

A pesar de que la actividad de los call centers es reciente, la rápida evolución en la tecnológica empleada y los cambios organizativos que la han acompañado, ha modificado sus características definitorias, con lo que se da paso a diversas formas de definirlo y comprenderlo. La definición de Pinilla (2005) es atinada en tanto sintetiza tres formulaciones que permiten tener mayor claridad de lo que entenderemos por ello. Al respecto, el autor señala que:

Los centros de llamadas se dedican casi en exclusiva a ofertar y cubrir múltiples $y$ variados servicios para clientes. Es el denominador común y el punto de partida de todos los centros de llamadas. Incluso cuando se encuentran incluidos en empresas industriales, el objetivo final de éste es de carácter comercial o de mejora de la calidad del servicio. En segundo lugar se da gran importancia al lugar, a la ubicación del centro de llamadas, centralizada para una mejor gestión, pero no sólo eso: en una zona bien comunicada, con servicios de nuevas tecnologías (actualmente se prima el ancho de banda), que pueda contar con un suministro elevado de trabajadores cualificados (Pinilla, 2005, p. 17). 
Este crecimiento queda especialmente reflejado en el empleo de la evolución tecnológica para la ampliación de servicios y su mejora. En un primer momento, la actividad ofrecía comunicación bidireccional, aunque solo de voz. En la actualidad, los avances en hardware, software y redes de comunicación son el motor de la evolución del centro de llamadas al centro de servicios compartidos que ofrecen mayores posibilidades en el campo de transmisión y procesamiento de datos o la estandarización de procesos. En este contexto, se desarrollarían los denominados "web-enabled call centers", los cuales integran la tecnología tradicional de los centros de llamadas con el empleo de Internet para complementar el servicio prestado.

Varios estudios realizados en Costa Rica, Brasil y Argentina señalan que quienes trabajan en call centers no planean que su estadía en esos lugares sea muy larga; por lo general, no más de dos años. Consideran al call center como una experiencia laboral que se espera terminar, por ejemplo, en el momento de obtener un título de educación superior que permita acceder a un empleo dentro de la propia área de estudio (Chacón, 2010; Rodríguez, 2008; Del Bono y Bulloni, 2008; Lerrer, 2008; Santos y Marques, 2006). Sin embargo, la noción de temporalidad con la cual inicialmente se incorporaban las personas —en su mayoría jóvenes - se ha modificado en los últimos años. Brown (2007) plantea que el establecimiento de los call center ha cambiado el perfil académico de sus empleados y empleadas, con el fin de asegurarse una mano de obra cualificada. Esto ha dado origen al desarrollo de programas de estudio desarrollados en el Instituto Nacional de Aprendizaje (INA), la Universidad Latinoamericana de Ciencia y Tecnología (ULACIT) y el Colegio Universitario Boston, que se dedican a mejorar el nivel de inglés y enseñan actividades de servicio al cliente para call center (Vargas, 2015).

El tema de la temporalidad de la actividad (el cual también se empieza a modificar), así como la juventud de quienes trabajan en estas empresas de servicio fueron aspectos que llamaron la atención y originaron estudios que captan dicha realidad. Autores como Sennett
(1999) y Crovi (2000) analizan la relación entre trabajos flexibles y personas jóvenes. Esta tesis es tomada en cuenta por Vargas (2015), quien realiza un estudio en Costa Rica y polemiza a partir de este enfoque. En su investigación, queda establecido que, en contraste con la percepción general que se tiene de estas personas, según la cual son principalmente jóvenes que utilizan el call center como un mecanismo de inserción temporal al mundo laboral, se identificaron muchas personas con perfiles diferentes al mencionado, tanto en términos etarios como con respecto a sus objetivos laborales. Igualmente, muchas personas llevan a cabo carreras laborales de muchos años en estos call centers, por lo que se cuestiona la noción del call center como "trabajo de paso". A su vez, a pesar de que la mayoría de las compañías cumplen con las garantías laborales mínimas estipuladas por la ley, se encontraron varias formas de disconformidad con las dinámicas laborales internas.

El trabajo de Vargas (2015) es, sin duda, un referente importante a nivel nacional que indaga temas de particular interés para nuestra investigación, puesto que analiza aspectos que aquí se proponen, como la valoración que tienen las personas sobre su trabajo, el nivel de satisfacción y el proyecto futuro.

\section{REFLEXIÓN FINAL}

Considerando aspectos teóricos y empíricos que se han ido planteando a lo largo del documento, surge la pregunta de si existen diferencias significativas en las condiciones de trabajo $y$ empleo entre quienes laboran en la industria textil y los call centers, y si las incidencias en la salud de dichas actividades económicas son similares o, por el contrario, muy disímiles. Si hay similitudes, ¿podemos explicar los factores que las acercan? El conocimiento empírico que existe acerca de la actividad de la industria textil (maquila) indica que las condiciones de empleo tienen un impacto diferencial en hombres y mujeres. ¿Sucede lo mismo con respecto a los call centers? Los estudios revisados al respecto no permiten identificar la existencia de estas diferencias.

Por otra parte, tomando en cuenta lo que indican los estudios sobre el mercado de trabajo 
$y$ las diferencias que existen entre hombres $y$ mujeres pautadas por aspectos estructurales del sistema socioeconómico y la discriminación vertical y horizontal que se da en el empleo, se pregunta sobre el valor, las expectativas $y$ el significado que le asigna cada quien a su trabajo. Asimismo, si se observan similitudes o diferencias con respecto a este, considerando el género y el sector de actividad, y si estas personas se sienten identificadas con la actividad que realizan, si se sienten parte de un colectivo o grupo social particular, o si se identifican con la empresa.

Finalmente, cabe señalar que se parte de la idea de que ambas actividades económicas - las maquilas textiles y los call centers- corresponden al proceso de apertura económica que se inicia en la segunda mitad de la década de los 80 en el país. Estas políticas fomentan una estrategia de desarrollo basada en la promoción de las exportaciones y la atracción de la inversión extranjera directa. La industria textil ligada al capital transnacional es una actividad que adquiere importancia y genera empleo a personas con bajo nivel educativo y experiencia laboral. Se ha caracterizado por emplear principalmente a las mujeres, ya que se considera que cuentan con más destrezas en cuanto al uso de las máquinas de coser y otras actividades propias de dicha actividad. Sin embargo, cabe notar que lo mismo ocurre en los call centers, donde las mujeres han empezado a tener una participación creciente.

El proceso de apertura ha continuado $y$ cambiado. Los call centers surgen en este contexto de globalización, donde se exacerba el uso de la información y la tecnología informática. Si bien, la industria textil hace uso de estos conocimientos, la lógica de producción sigue siendo la misma en lo que respecta al ensamblaje $y$, en algunos casos, hacen uso de servicios tercerizados para bajar costos de producción. Así, a finales del siglo xx, se experimentó un crecimiento en "la extensión de la producción inmaterial y de la transformación en objetos simbólicos" (de la Garza, 2005, p. 13).

Los call centers se gestan en Costa Rica en la década de los 90 y han tenido un proceso de desarrollo desigual. En la primera década del siglo xxI, presentaron un abrupto crecimiento, el cual ha seguido aumentando. Este crecimiento se ha dado a pesar de las proyecciones que se hicieron, ya que se creía que muchas empresas dejarían el país por los costos de producción, mano de obra e impuestos que deben pagar. Pero eso no ha pasado; en cambio, hay empresas que, desde su llegada al país, no dejan de expandirse, crecer $y$ ofrecer nuevos servicios cada vez más complejos (Piedra, 2019).

Por lo tanto, se afirma que las condiciones de empleo y trabajo en ambos sectores son similares; en ambos casos, se considera que son espacios laborales que presentan características neo-tayloristas como "la sumisión al tiempo $y$ la estructura de los softwares, el control racional del tiempo y del trabajo por medio de la informática; la productividad máxima en detrimento de las buenas condiciones físicas y psicológicas de los trabajadores" (Lerrer, 2008, p. 7). Por ello se afirma que los call centers son la "maquila" del siglo xxi, así como la industria textil. En el caso de los call centers, se ha constatado que genera altos niveles de estrés y efectos en la salud, son cuerpos que sufren por la intensidad y rigurosidad con que se trabaja, a pesar de que cuentan con condiciones de empleo formales $y$ en su mayoría aceptables, con flexibilidad en el uso del tiempo y los turnos (Piedra, 2019).

Asimismo, se señala que, tanto la industria textil transnacional como los calls centers, representan la maquila de la nueva organización del trabajo. Además, a pesar de que cada cual realiza actividades diametralmente distintas - una productiva y la otra de servicios-ambas persisten más allá del tiempo y el espacio de forma paralela, readecuándose a nuevos requerimientos del mercado.

\section{REFERENCIAS}

Aglietta, M. (1976). Regulación y crisis del capitalismo. Siglo XXI Editores.

Antunes, R. (2003). ¿Adiós al trabajo?. Herramienta, $2^{\text {a }}$ ed.

Antunes, R. (2015). Los modos de ser de la informalidad: ¿hacia una nueva era de la precarización estructural del trabajo? Ecuador Debate, 94, 69-79. 
Bauman, Z. (2004). Modernidad líquida, $4^{\mathrm{a}}$ reimpr. Fondo de Cultura Económica.

Benach, J. y Muntaner, C. (2005). Aprender a mirar la salud, cómo la desigualdad social daña nuestra salud. España, el Viejo Topo.

Benach, J. y Muntaner, C. (2009). La epidemia global de desigualdad en salud tiene su origen en la crisis socio-ecológica del capitalismo. Revista Ecología Política, 37.

Boyer, R. (1989). La teoría de la regulación: un análisis crítico. Editorial Humanitas.

Boyer, R. (1996). Los orígenes de la teoría de la regulación. En Boyer, R. y Saillard, Y. (coord.). Teoría de la regulación: estado de los conocimientos. $\mathrm{CBC}$.

Boyer R. y Freyssenet M. (2003). Los modelos productivos. Editorial Fundamentos.

Braverman, H. (1974). Trabajo y capital monopolista. Nuestro Tiempo.

Brown, P. (2007). Implicaciones de los Call Centers en el ámbito del derecho. [Tesis para optar por el título de Licenciado en Derecho]. Universidad de Costa Rica.

Carrillo, F. (1999). Estudios de identificación de necesidades de empresarios de la maquila en Costa Rica. Informe de investigación, San José, oIT.

Carrillo, O. M. (1998). Zona Franca y mujer en Costa Rica. Liga Internacional de Mujeres Pro Paz y Libertad Comité de Mujer Trabajadora.

Cordero, A. (1999). ¿Qué ha pasado con la maquila? Actualización del estudio sobre la situación laboral en las empresas maquiladoras del Istmo Centroamericano y República Dominicana. Seminario Sub-regional para el Istmo Centroamericano, República Dominicana y Panamá sobre "Relaciones laborales y condiciones de trabajo en las ZPE y empresas maquiladoras". San José, Costa Rica.

Chacón, M. (2010). Apostando al siglo xxi: El desarrollo de los "Sportbooks" y las representaciones de trabajo, proyecto de vida y consumo de las y los jóvenes trabajadores. [Tesis para optar por el título de Licenciado en Estudios
Latinoamericanos]. Universidad Nacional. Heredia, Costa Rica.

Crovi Druetta, D. (2000). Los Jóvenes ante la convergencia tecnológica: ¿integración o 259 exclusión? Comunicación y Sociedad, 38, 127-143. http://www.publicaciones.cucsh.udg.mx/pperiod/comsoc/ pdf/38_2000/127-143.pdf

Delclós, G., Piedra N. y otros. (2012). I Encuesta Centroamericana sobre Condiciones de Trabajo y Salud (IECCTS). Informe general estudio cualitativo basado en el análisis de grupos focales. Barcelona, CISAL.

De la Garza, E. (2005). Del concepto ampliado de trabajo al de sujeto laboral ampliado. CLACSO.

De la Garza, E. (junio de 2007). Trabajo y la vida a comienzo del siglo XXI. Ponencia presentada en el seminario en la Universidad Complutense de Madrid: Los Estudios Laborales en América Latina al inicio del siglo Xxi, España.

De la Garza, E. (2010). Hacia un concepto ampliado de trabajo. Del concepto clásico al no clásico. Anthropos.

De la Garza, E. (editor). (2016). Los estudios laborales en América Latina: Orígenes, desarrollo y perspectivas. Anthropos Editorial, España y Universidad Autónoma Metropolitana-Iztapalapa, México.

De La Garza, H. (2005). Neoinstitucionalismo ¿alternativa a la Elección Racional?: una discusión entre economía y la sociología. Nuevos enfoques teóricos y estudios laborales. Rev. Mex. Sociología, 67 (1), 29-65.

De la Garza, H. (2015). Estado de la literatura del trabajo en América Latina en los últimos 30 años. Exposición presentada en el Congreso Latino Americano de Sociología ALAS-Costa Rica.

Del Bono, A. y Bulloni, M. (2008). Experiencias laborales juveniles: Los agentes telefónicos de call centers offshore en Argentina. Trabajo y Sociedad (Revista electrónica), IX (10). http://www.unse.edu.ar/trabajoysociedad/DEL_BONO.pdf

Dunlop, J. (1957). La tarea de la teoría salarial contemporánea. en G. Taylor y F. Pierson (eds.), Nuevos conceptos en la discriminación salarial (pp. 117-139). McGraw-Hill. 
Freedman, G. (1992). The Anatomy of Work. Transactions Publishers.

Gitli, E. (1997). La industria de la maquila en Centroamérica. Oficina Internacional de Trabajo, San José, oIT.

Goldthorpe, J. (1989). Ordine e Conflicto nel Capitalismo Moderno. Il Mulino.

Grijalva Monteverde, G. y Covarrubias Valdenebro, Á. (coords.) (2005). Las mujeres en la maquila. Trabajo, instituciones de apoyo y organización sindical. Sociedad, XVII (32), 189-203.

Hyman, R. (1987). Strategy or Structure? Capital, Labour and Control. Work Employment and Society, 1.

Hochschild, A. R. $(2007 \mathrm{a})$. The Commercialization of Intimate life: Notes from Home and Work. University of California Press.

Hochschild, A. R. (2007b). Through the Crack of the Time Bind: From Market Management to Family Management. Anthropology of Work Review, XxVIII, 1-8.

Hochschild, A. R. (2008). Feeling Around the World. Contex. http://contexts.org/articles/ spring-2008/feeling-around-the-world

Kern, H. y Schumann, M. (1984). Work and Social Character: Old and New Contours. SAGE.

Kerr, H. y Schumann, M. (1998). Limits of the Division of Labour: New Production and Employment Concepts in West Germany Industry. British Journal of Industrial Relations, 33 (3).

Lerrer, C. (2008). Construção da identidade no trabalho em call centers: a identidade provisória. Red de Revistas Científicas de América Latina y el Caribe, España y Portugal. http://redalyc.uaemex.mx/redalyc/src/inicio/ArtPdfRed. jsp?iCve $=46140313$

Lipietz, A. (1983). El Capital y su espacio. Siglo XXI.

Lipietz, A. (1992). Hacia una mundialización del fordismo? Teoría y politica, 7 .

Mora, M. (2010). Ajuste y empleo. La precarización del trabajo asalariado en la era de la globalización. El Colegio de México.

Mora, M. (2011). El empleo precario asalariado y globalización: enseñanzas desde
Costa Rica. En: Pacheco, de la Garza y Reygadas (coords.), Trabajos atipicos y precarización del empleo. El Colegio de México, Centro de Estudios Demográficos, Urbanos y Ambientales.

Naville, P. (1985). Hacia el automatismo social: problemas del trabajo y de la automatización. Fondo de Cultura Económica.

Neffa. J. (2005). Evolución conceptual de la teoría de la regulación. file:///D:/ Us ers/31635/G o ogle\% 20 Drive/ BIBLIOTECA\%20NANCY/Trabajo/lateoradelaregulacin.pdf

Neffa, J. (2010). La transición desde los "verdaderos empleos" al trabajo precario. En: Enrique de la Garza y Julio C. Neffa (coords.), Trabajo, identidad y acción colectiva (43-80). Universidad Autónoma Metropolitana (UAM) Plaza y Valdez.

Neffa, J. y de la Garza, E. (s.f). Trabajo y Modelos de Producción en América Latina. Modelos Económicos, Modelos Productivos y Estrategias de Ganancia: Concepto y Problematización.

Negri, A. (1978). Del obrero-masa al obrero social. Tecnos.

Pacheco, E. y de la Garza, E. (2011). Trabajos atípicos y precarización del empleo. El Colegio de México. Centro de Estudios Demográficos, Urbanos y Ambientales.

Panzieri, R. (1978). Acerca del uso capitalista de la máquina. La división capitalista del Trabajo. Siglo XxI.

Piore, M. y Charles, S. (1990). La segunda ruptura industrial. Alianza.

Pérez Sáinz, J. P. (1995a). Actores y cultura laboral en la industria de exportación: una comparación entre Costa Rica y la República Dominicana. Oficina Internacional del Trabajo.

Pérez Sáinz, J. P. (1995b). Impacto sociolaboral de maquilas y zonas francas en Centroamérica y República Dominicana. Oficina Internacional del Trabajo.

Pérez Sáinz, J. P. (1998). Ciudananía Social y derechos laborales en Centroamérica. Cuadernos de Ciencias Sociales, 180. Sede Académica Flacso Costa Rica. 
Pérez Sáinz, J. P. (2015). Mercados y bárbaros. La persistencia de las desigualdades de excedente en América Latina. FlACso, Costa Rica.

Pérez Sáinz, J. P. y Cordero, A. (1996). Los nuevos escenarios laborales en Centroamérica una propuesta de análisis. Anuario de Estudios Centroamericanos, 20 (2).

Pérez, C. y Ominami. C. (comp.) (1985). La tercera revolución tecnológica. Gel.

Piedra, N. (2000). Entre hilos y telas: Primer diagnóstico de las condiciones laborales y de vida de las trabajadoras de la maquila. OIT.

Piedra, N. (2019). El trabajo en los centers: la temporalidad y la corporeidad de la experiencia laboral. Revista Rupturas, 10 (1), 103-137. https://doi.org/10.22458/ rr.v10i1.2752

Pinilla, J. (2005). 2. Los centros de llamadas en España, estudio cualitativo de la Encuentra de Trabajo y Salud. España.

Quintero, C. (1990). La sindicalización en las maquiladoras tijuanenses: 1970-1988. Consejo Nacional para la Cultura y las Artes.

Quintero C. (1997). Reestructuración sindical en la frontera Norte. El caso de la industria maquiladora. 1 era. Edición. El Colegio de la Frontera Norte, México.

Quintero, C. (2013). Sindicatos y regulación del mercado laboral femenino en maquiladoras. La experiencia de Matamoros, Tamaulipas. http://www.mriuc.bc.uc.edu. ve/handle/123456789/1045

Quintero, C. y Dragustinovis, J. (2006). Soy más que mis manos. Los diferentes mundos de la mujer en la maquila. 1 era. Edición. Fundación Friedrich Ebert/Sindicato de Jornaleros y Obreros Industriales y de la Industria Maquiladora
Rodríguez, L. (2008). Juventud y cambio social en Costa Rica: trabajadores de call centers y Sportbooks. Ejercicio empírico desde una perspectiva etnográfica. Revista Estudios, 21, 271-289.

Santos, M. J. y Marques, A. P. (2006). O Caso dos Call Centers: Organização do trabalho e atitudes face ao trabalho e emprego. Sociologia, 52, 67-86. http://www.scielo. oces.mctes.pt/pdf/spp/n52/n52a04.pdf

Sennett, R. (1999). A Corrosão do Caráter. (Trad. M. Santarrita). Rio de Janeiro, Editora Record. (Original en inglés, 1998).

Trejos, M. E. y Abdallah, L. (2000). Discusión y aplicación de la flexibilidad laboral en Centroamérica. Espacios Consultores.

Touraine, A. (1973). La sociedad post-industrial. Editorial Ariel.

Vargas, G. (2015). Representaciones sociales en torno al trabajo: procesos de construcción identitaria, realización laboral $y$ transitoriedad en las $y$ los trabajadores de call center en Costa Rica. [Tesis para optar por el grado de Licenciatura en Sociología]. Universidad de Costa Rica.

Zapata, F. (2010). Desentrañar el sentido del trabajo. Hacia la comprensión de las configuraciones identitarias laborales. El Colegio de México.

Zúñiga, M. (2005). Reconfiguración laboral, flexibilidad y violencia de género. Memorias de evento internacional Dominación y resistencia. México, UAM-Xochimilco.

Fecha de ingreso: 01/06/2020 Fecha de aprobación: 14/10/2021 
